\title{
ON GENERALIZED BRAID GROUPS
}

\author{
by A. K. NAPTHINE and STEPHEN J. PRIDE
}

(Received 9 July, 1985)

1. Introduction. Braid groups were introduced by Artin [1]. These groups have been studied extensively-see [2], [9] and the references cited there. Recently work has been done on "circular" braid groups and other "braid-like" groups [7], [10]. In this paper we formulate the concept of a generalized braid group, and we begin a study of the structure of such groups. In particular for such a group $G$, there is a homomorphism from $G$ onto the infinite cyclic group, the kernel of which is the derived group $G^{\prime}$ of $G$. We study $G^{\prime}$. Our results generalize results of Gorin and $\operatorname{Lin}[5]$, who considered the case when $G$ is a classical braid group $B_{n}(n \geqslant 3)$. They showed that $B_{n}^{\prime} / B_{n}^{\prime \prime}$ is free abelian of rank 2 if $n=3,4$ and is trivial if $n \geqslant 5$. They also showed that $B_{n}^{\prime}$ is finitely presented.

Our methods involve a rather geometric version of combinatorial group theory. We have collected all the material needed into an Appendix, which the reader should refer to for any unfamiliar terms or concepts. The Appendix is, of necessity, somewhat condensed. Further details can be found in the books [4], [8], [12], [13], [14], or in a forthcoming article [11] by the second author. As well as the material in the Appendix, we will need a small amount of (very standard) graph theory. For this, we refer the reader to $[3]$.

Let $\Gamma$ be a finite graph with vertex set $\mathbf{x}$, and suppose that some edges of $\Gamma$ are coloured red and the rest are coloured green. We assume that:

any two vertices of $\Gamma$ are joined by a path consisting entirely of green edges.

We associate with the edge-coloured graph $\Gamma$ a presentation

$$
\mathscr{K}=\langle\mathscr{X} ; \beta(x, y)(\{x, y\} \text { an edge of } \Gamma)\rangle,
$$

where $\mathscr{X}$ is the bouquet with edges $x, x^{-1}(x \in \mathbf{x})$, and where

$$
\beta(x, y)= \begin{cases}(x y x)(y x y)^{-1} & \text { if }\{x, y\} \text { is a green edge, } \\ (x y)(y x)^{-1} & \text { if }\{x, y\} \text { is a red edge. }\end{cases}
$$

The fundamental group $\pi_{1}(\mathscr{X})$ is a generalized braid group.

Note that $\beta(y, x)$ is equal to $\beta(x, y)^{-1}$, so as far as $\pi_{1}(\mathscr{K})$ is concerned, there is actually no real need to include both $\beta(x, y)$ and $\beta(y, x)$. However, for technical reasons it is convenient to do so.

We remark that the classical braid group $B_{n}$ is obtained by taking $\Gamma$ to be the edge-coloured complete graph $K_{n-1}$, with vertices $a_{1}, \ldots, a_{n-1}$, green edges $\left\{a_{1}, a_{2}\right\}, \ldots,\left\{a_{n-2}, a_{n-1}\right\}$, and all other edges red.

There is a homomorphism from $\pi_{1}(\mathscr{K})$ onto $\mathbb{Z}$ which takes $[x]$ to 1 for each $x \in \mathbf{x}$. Using (*), it is not difficult to show that the kernel of this homomorphism is $\pi_{1}(\mathscr{K})^{\prime}$. Our aim in this paper is to study the group $\pi_{1}(\mathscr{K})^{\prime}$.

Glasgow Math. J. 28 (1986) 199-209. 
Consider the subgraph $\Gamma_{\text {red }}$ of $\Gamma$ consisting of all the vertices of $\Gamma$, together with all the red edges. Suppose this graph has $r$ components with vertex sets $\mathbf{x}_{1}, \mathbf{x}_{2}, \ldots, \mathbf{x}_{r}$. We let $\Gamma_{m}$ denote the induced subgraph of $\Gamma$ on the vertex set $\mathbf{x}_{m}(m=1, \ldots, r)$. We let $\bar{\Gamma}$ denote the graph obtained from $\Gamma$ by identifying all elements of $\mathbf{x}_{m}$ to a single vertex $m$ $(m=1,2, \ldots, r)$. Note that by $(*), \overline{\bar{\Gamma}}$ is connected.

TheOREM 1. The abelianization $\left(\pi_{1}(\mathscr{K})^{\prime}\right)^{\mathrm{ab}}$ of $\pi_{1}(\mathscr{K})^{\prime}$ is free abelian of rank $2(r-1)$.

THEOREM 2. The group $\pi_{1}(\mathscr{K})^{\prime}$ is finitely presented if the following conditions hold: each $\Gamma_{m}(1 \leqslant m \leqslant r)$ is a complete graph; $\bar{\Gamma}$ is a tree; if $\{m, n\}$ is an edge of $\bar{\Gamma}$ then $\{x, y\}$ is an edge of $\Gamma$ for all $x \in \mathbf{x}_{m}, y \in \mathbf{x}_{n}$.

Observe that when $\Gamma$ is the edge-coloured graph $K_{n-1}$ described above, then $\Gamma$ satisfies the conditions of Theorem 2 .

These theorems will be proved by considering the covering $\tilde{\mathscr{K}}$ of $\mathscr{K}$ corresponding to $\pi_{1}(\mathscr{K})^{\prime}$. The 1 -skeleton of $\mathscr{\mathscr { K }}$ has vertex set $\mathbb{Z}$ and edge set $\left\{x_{i}^{ \pm 1}: x \in \mathbf{x}, i \in \mathbb{Z}\right\}$, where $\iota\left(x_{i}\right)=i, \tau\left(x_{i}\right)=i+1$. The defining paths are indexed by ordered triples $(x, y, i)$ where $\{x, y\}$ is an edge of $\Gamma$, and $i \in \mathbb{Z}$. The defining path $\beta(x, y, i)$ corresponding to $(x, y, i)$ is given by:

$$
\begin{array}{ll}
\left(x_{i} y_{i+1} x_{i+2}\right)\left(y_{i} x_{i+1} y_{i+2}\right)^{-1} & \text { if }\{x, y\} \text { is a green edge, } \\
\left(x_{i} y_{i+1}\right)\left(y_{i} x_{i+1}\right)^{-1} & \text { if }\{x, y\} \text { is a red edge. }
\end{array}
$$

The following terminology will be useful for the proof of Theorem 2: $x_{i}$ (resp. $y_{i}^{-1}$ ) will be called the minimum $x$-edge (resp. $y$-edge) in $\beta(x, y, i) ; x_{i+2}$ (resp. $y_{i+2}^{-1}$ ) will be called the maximum $x$-edge (resp. $y$-edge) in $\beta(x, y, i)$ if $\{x, y\}$ is green, while $x_{i+1}$ (resp. $y_{i+1}^{-1}$ ) will be called the maximum $x$-edge (resp. $y$-edge) if $\{x, y\}$ is red.

We will need the following notation: $b(m)$ will denote a fixed but arbitrary element of $\mathbf{x}_{m}(m=1,2, \ldots, r)$, and $\mathbf{b}$ will denote the set $\{b(m): 1 \leqslant m \leqslant r\}$; for $x \in \mathbf{x}_{m}$ we define $b^{(x)}$ to be $b(m)(1 \leqslant m \leqslant r)$.

ACKNowledgement. The authors thank R. J. Steiner for indicating how to simplify our original homology calculation in the proof of Theorem 1 . We also thank the referee for comments which improved the Introduction.

2. Proof of Theorem 1. The group $\left(\pi_{1}(\mathscr{K})^{\prime}\right)^{\mathrm{ab}}$ is isomorphic to the first integral homology group $H_{1}=H_{1}(\tilde{\mathscr{K}} ; \mathbb{Z})$. We determine the structure of $H_{1}$.

First observe that the group $Z_{1}$ of 1-cycles is generated by

$$
\left\{x_{i}-y_{i}: x, y \in \mathbf{x}, i \in \mathbb{Z}\right\} .
$$

For let $c=\varepsilon_{1} e_{1}+\varepsilon_{2} e_{2}+\ldots+\varepsilon_{l} e_{l}$ be a 1-cycle. Here each $\varepsilon_{\lambda}$ is \pm 1 , and each $e_{\lambda}$ is an element of $\left\{x_{i}: x \in \mathbf{x}, i \in \mathbb{Z}\right\}$. Thus each $e_{\lambda}$ is an element of $\mathbf{x}$ with a subscript. Suppose $k$ is the highest subscript which occurs. Then one of the terms of $c$ has the form $\varepsilon x_{k}$ for some $x \in \mathbf{x}$. Now the only terms of $c$ which contribute to the coefficient of $k+1$ in $\partial(c)$ are those with subscript $k$. Since the coefficient of $k+1$ in $\partial(c)$ is 0 , there must be a term 
$-\varepsilon y_{k}$ with $y \in \mathbf{x}$. Then $c=\varepsilon\left(x_{k}-y_{k}\right)+c^{\prime}$, where $c^{\prime}$ is a 1 -cycle with fewer terms than $c$. Now use induction. relations

Let $S_{m}(1 \leqslant m \leqslant r)$ be the abelian group with generators $s(m)_{i}(i \in \mathbb{Z})$ and defining

$$
s(m)_{i}-s(m)_{i+1}+s(m)_{i+2}=0 \quad(i \in \mathbb{Z}),
$$

and let $S=\bigoplus_{m} S_{m}$. Note that $S_{m}$ is free abelian of rank 2 (with free basis $\left.s(m)_{0}, s(m)_{1}\right)$, and so $S$ is free abelian.

There is a homomorphism from the group $C_{1}$ of 1-chains onto $S$ defined by:

$$
x_{i} \mapsto s(m)_{i}\left(x \in \mathbf{x}_{m}, m=1, \ldots, r, i \in \mathbb{Z}\right) .
$$

The image of the group $B_{1}$ of 1-boundaries under this homomorphism is 0 . The image of $Z_{1}$ is $\operatorname{sgp}\left\{s(m)_{i}-s(n)_{i}: 1 \leqslant m<n \leqslant r, i \in \mathbb{Z}\right\}$, which has free basis

$$
\mathrm{s}=\left\{s(m)_{0}-s(1)_{0}, s(m)_{1}-s(1)_{1}: 2 \leqslant m \leqslant r\right\} .
$$

There is thus a homomorphism $\phi$ from $Z_{1} / B_{1}\left(=H_{1}\right)$ onto sgps induced by the homomorphism $C_{1} \rightarrow S$. We show that $\phi$ is an isomorphism.

Define a homomorphism $\theta: \operatorname{sgp~} s \rightarrow Z_{1} / B_{1}$ by

$$
s(m)_{j}-s(1)_{j} \mapsto b(m)_{j}-b(1)_{j}+B_{1} \quad(2 \leqslant m \leqslant r, j=0,1) .
$$

Clearly $\phi \theta$ is the identity on sgp s. Also, $\theta \phi$ fixes the elements

$$
b(m)_{j}-b(n)_{j}+B_{1} \quad(m, n=1,2, \ldots, r ; j=0,1) .
$$

Consequently $\theta \phi$ will be the identity, provided the elements in (1) generate $Z_{1} / B_{1}$. We now show that this is the case.

Suppose $x, y \in \mathbf{x}$. Choose a path in $\Gamma$ from $x$ to $y$ consisting of green edges, and let the successive vertices of the path be $x={ }^{0} x,{ }^{1} x,{ }^{2} x, \ldots,{ }^{\prime} x=y$. Then for $i \in \mathbb{Z}$,

$$
\left(x_{i}-y_{i}\right)-\left(x_{i+1}-y_{i+1}\right)+\left(x_{i+2}-y_{i+2}\right)=\sum_{\mu=0}^{l-1} \partial \beta\left({ }^{\mu} x,{ }^{\mu+1} x, i\right) \in B_{1} .
$$

Suppose $t, z \in \mathbf{x}_{m}$ for some $m$. By considering a path in $\Gamma$ from $t$ to $z$ consisting of red edges we show, in a similar way to the above, that for $i \in \mathbb{Z}$

$$
\left(t_{i+1}-z_{i+1}\right)-\left(t_{i+2}-z_{i+2}\right) \in B_{1} .
$$

Using (2), it then follows that

$$
t_{i}-z_{i} \in B_{1} \text {. }
$$

Now using (2) it is easily shown that $Z_{1} / B_{1}$ is generated by the elements

$$
x_{j}-y_{j}+B_{1} \quad(x, y \in \mathbf{x}, j=0,1) \text {. }
$$

But (3) implies that

$$
x_{j}-y_{j}+B_{1}=b_{j}^{(x)}-b_{j}^{(y)}+B_{1},
$$


since

$$
x_{j}-y_{j}=\left(x_{j}-b_{j}^{(x)}\right)+\left(b_{j}^{(x)}-b_{j}^{(y)}\right)+\left(b_{j}^{(y)}-y_{j}\right) .
$$

Hence the elements in (1) generate $Z_{1} / B_{1}$, as required.

3. Proof of Theorem 2. We choose a fixed but arbitrary spanning subforest $\Phi$ of $\Gamma_{\text {red. }}$. For $x \in \mathbf{x}$ we define $d(x)$ to be the length of the (unique) path in $\Phi$ from $b^{(x)}$ to $x$. For $\{x, y\}$ an edge of $\Gamma$, we define wt $\{x, y\}$ to be $d(x)+d(y)$.

Lemma 1. Let $\{m, n\}$ be an edge of $\bar{\Gamma}$. If $x \in \mathbf{x}_{m}, y \in \mathbf{x}_{n}, j \in \mathbb{Z}$ then $\beta(x, y, j)$ is a consequence of

$$
\begin{aligned}
& \beta(s, t, 0), \beta(s, t, 1), \beta(s, t, 2) \quad\left(s \in \mathbf{x}_{m}, t \in \mathbf{x}_{n}\right), \\
& \beta(b(m), b(n), i) \quad(i \in \mathbb{Z}), \\
& \beta(s, t, i) \quad(\{s, t\} \text { a red edge, } i \in \mathbb{Z}) .
\end{aligned}
$$

Proof. By induction on wt $\{x, y\}$. If $\mathrm{wt}\{x, y\}=0$ there is nothing to prove.

Suppose wt $\{x, y\}>0$. We may assume that $d(x)>0$. Let $z$ be the penultimate vertex of the path in $\Phi$ from $b(m)$ to $x$. Now we deduce from the van Kampen diagram in Figure 1 :

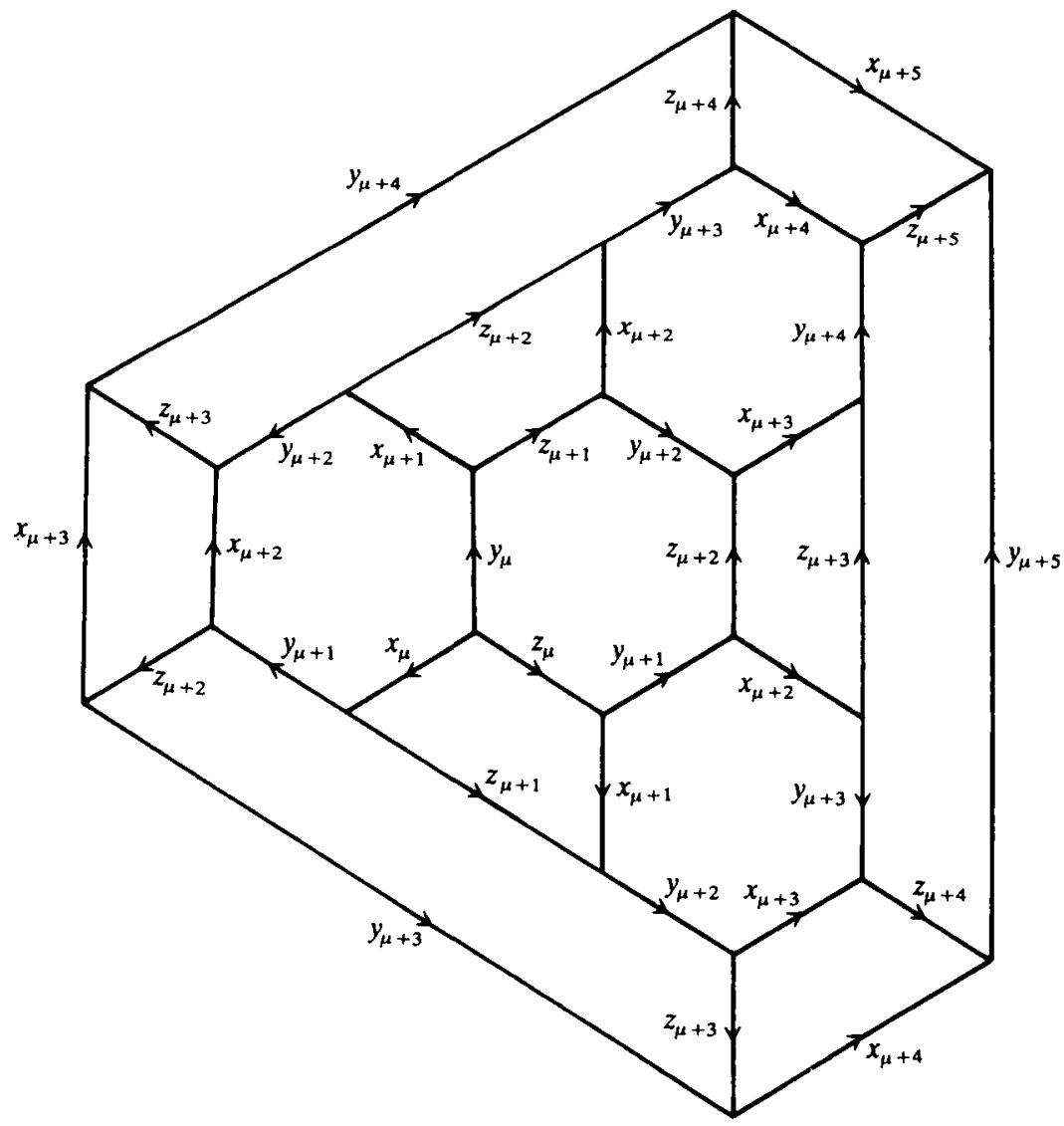

Figure 1 
that for any $\mu \in \mathbb{Z}, \beta(x, y, \mu+3)$ is a consequence of

$$
\begin{aligned}
& \beta(x, y, \mu), \beta(x, y, \mu+1), \beta(x, y, \mu+2), \\
& \beta(z, y, i) \quad(i \in \mathbb{Z}), \\
& \beta(s, t, i) \quad(\{s, t\} \text { a red edge, } i \in \mathbb{Z}) .
\end{aligned}
$$

Also, $\beta(x, y, \mu)$ is a consequence of

$$
\beta(x, y, \mu+1), \beta(x, y, \mu+2), \beta(x, y, \mu+3),
$$

together with the defining paths (4), (5). It follows (by an easy induction on $|j|)$ that $\beta(x, y, j)$ is a consequence of

$$
\beta(x, y, 0), \beta(x, y, 1), \beta(x, y, 2)
$$

and the paths (4), (5).

Now since wt $\{z, y\}<\operatorname{wt}\{x, y\}$, our inductive hypothesis applies to the paths (4), and the lemma follows.

Lemma 2. If $x, y \in \mathbf{x}_{m}(1 \leqslant m \leqslant r, x \neq y)$ and $j \in \mathbb{Z}$, then $\beta(x, y, j)$ is a consequence of the paths

$$
\beta(s, b(m), i) \quad\left(s \in \mathbf{x}_{m}, s \neq b(m), i \in \mathbb{Z}\right),
$$

together with the paths

$$
\beta(s, t, i) \quad\left(s, t \in \mathbf{x}_{m}, s \neq t\right),
$$

where $i \in\{0,1\}$ if $\{s, t\}$ is a red edge and $i \in\{0,1,2\}$ if $\{s, t\}$ is a green edge.

Proof. By induction on wt $\{x, y\}$. If wt $\{x, y\}=1$, or more generally, if one of $x, y$ is $b(m)$, then the result holds trivially. Thus, for the induction step we may assume that $0<d(x) \leqslant d(y)$. Let $z$ be the penultimate vertex of the path in $\Phi$ from $b(m)$ to $x$. Note that

$$
\mathrm{wt}\{z, x\}=d(z)+d(x)=(d(x)-1)+d(x)<\mathrm{wt}\{x, y\} .
$$

Note also that $\mathrm{wt}\{z, y\}<\mathrm{wt}\{x, y\}$.

Suppose $\{x, y\}$ is a green edge. Then using Figure 1 (if $\{z, y\}$ is a green edge), or Figure 2 (if $\{z, y\}$ is a red edge), we find, using arguments like those in the proof of Lemma 1 , that in both cases $\beta(x, y, j)$ is a consequence of (at most)

$$
\begin{aligned}
& \beta(x, y, 0), \beta(x, y, 1), \beta(x, y, 2), \\
& \beta(z, y, i) \quad(i \in \mathbb{Z}), \\
& \beta(z, x, i) \quad(i \in \mathbb{Z}) .
\end{aligned}
$$

On the other hand, if $\{x, y\}$ is a red edge then we find, using Figure 3 (if $\{z, y\}$ is a green edge), or Figure 4 (if $\{z, y\}$ is a red edge) that $\beta(x, y, j)$ is a consequence of (at most) 


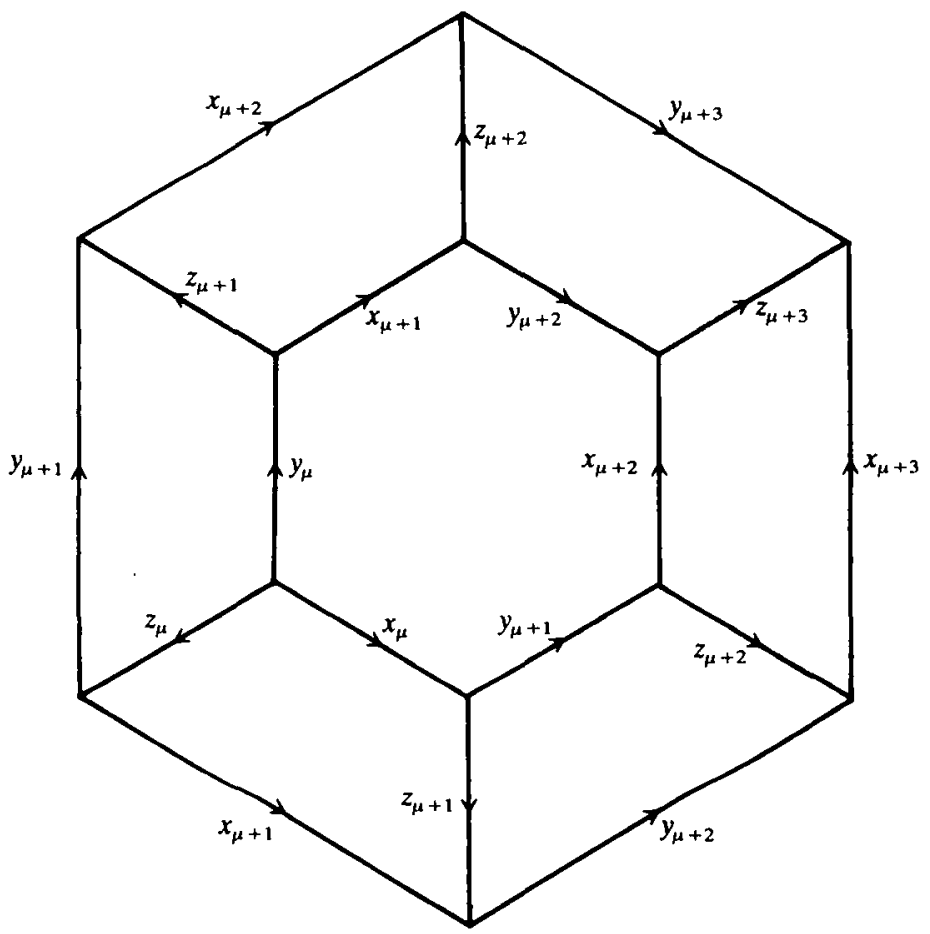

Figure 2

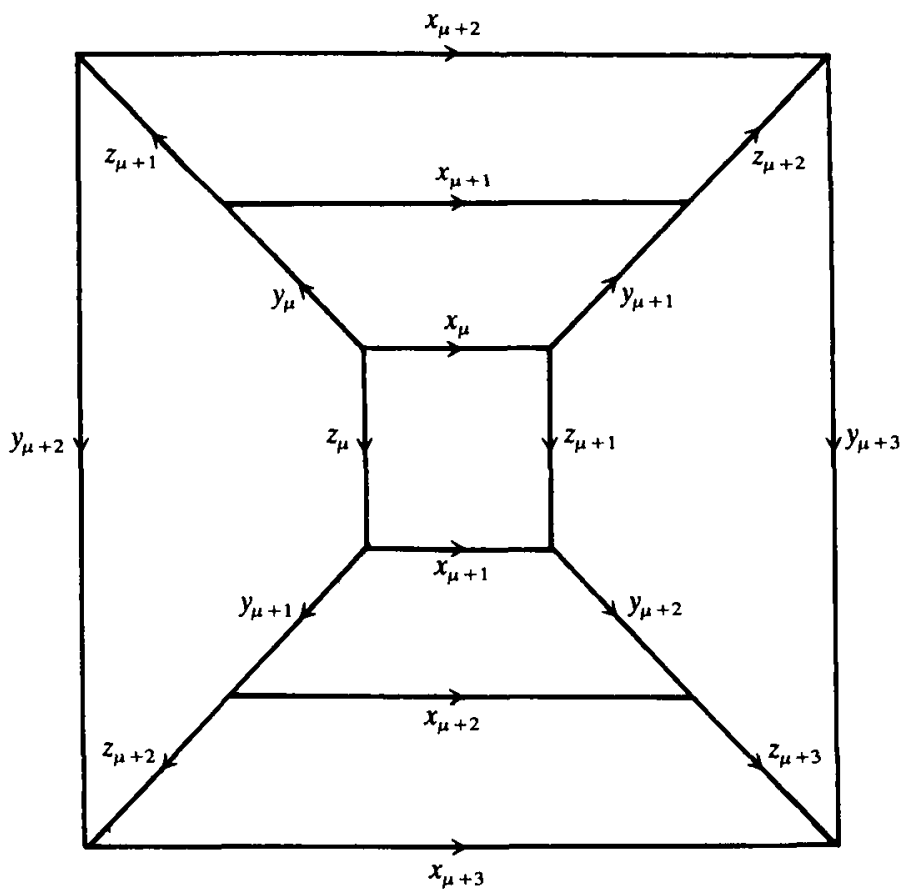

Figure 3 


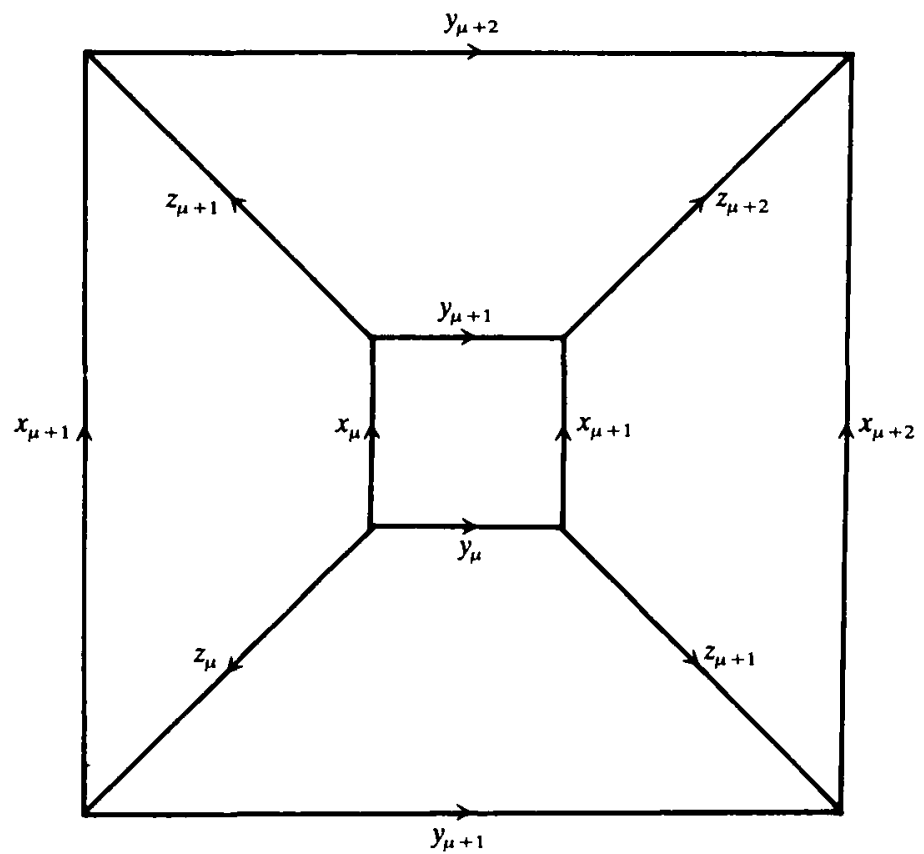

Figure 4

$$
\beta(x, y, 0), \beta(x, y, 1)
$$

and the paths (6), (7); now applying the inductive hypothesis to the paths (6), (7) gives the result.

We deduce from Lemmas 1 and 2 that $\pi_{1}(\tilde{\mathscr{K}})=\pi_{1}(\mathscr{L})$, where $\mathscr{L}$ is the complex with the same 1 -skeleton as $\tilde{\mathscr{K}}$ and with defining paths $\beta(\lambda)$, where $\lambda$ ranges over the elements

$$
\begin{gathered}
(s, t, 0),(s, t, 1),(s, t, 2) \quad(\{s, t\} \text { a green edge }), \\
(s, t, 0),(s, t, 1) \quad(\{s, t\} \text { a red edge }), \\
(b(m), b(n), i) \quad(1 \leqslant m<n \leqslant r,\{m, n\} \text { an edge of } \bar{\Gamma}, i \in \mathbb{Z}), \\
\left(s, b^{(s)}, i\right) \quad(s \in \mathbf{x}-\mathbf{b}, i \in \mathbb{Z}) .
\end{gathered}
$$

To proceed further, we need the following general result.

Let $\mathcal{M}=\langle\mathscr{Y} ; \beta(\lambda)(\lambda \in \Lambda)\rangle$. Suppose that $\Lambda$ is expressed as a disjoint union

$$
\Lambda=\bigcup_{l=0}^{\infty} \Lambda_{l}
$$

An element of $\Lambda_{l}$ will be said to be of level l. Assume that the following is satisfied: if $\lambda$ has level $l>0$, then some cyclic permutation of $\beta(\lambda)$ has the form $e(\lambda) \alpha(\lambda)$, where $e(\lambda)$ is an edge, and $e(\lambda)^{ \pm 1}$ does not occur in $\alpha(\lambda)$ or in any $\beta(\mu)(\mu \neq \lambda)$ with $\mu$ of level $k$ 
$1 \leqslant k \leqslant l$. We call $e(\lambda)$ the edge corresponding to $\lambda$. Let

$$
\mathscr{Y}_{0}=\mathscr{Y}-\left\{e(\lambda)^{ \pm 1}: \lambda \text { has level greater than } 0\right\} .
$$

Then there are closed paths $\gamma(\lambda)\left(\lambda \in \Lambda_{0}\right)$ in $\mathscr{Y}_{0}$ such that $\left\langle\mathscr{Y}_{0} ; \gamma(\lambda)\left(\lambda \in \Lambda_{0}\right)\right\rangle$ is equivalent to $M$ : moreover, $\gamma(\lambda)=\beta(\lambda)$ if $\beta(\lambda)$ is a path in $\mathscr{Y}_{0}$. A proof of this result, together with further applications (for example, to surfaces), can be found in [11]. The result can be stated more precisely, but the above formulation is adequate for present purposes. (However, if one wants to use the proof of Theorem 2 given here to obtain an explicit finite presentation for $\pi_{1}(\mathscr{K})^{\prime}$, then a more precise version of the above result, giving details of the $\gamma(\lambda)$ 's, is needed.)

We now obtain a succession of equivalences of $\mathscr{L}$.

Define an index $\lambda$ of $\mathscr{L}$ to have:

level 0 if $\lambda$ is as in (8), (9) or (10);

level $l(l>0)$ if $\lambda=\left(s, b^{(s)}, \varepsilon(l-1)\right), s \in \mathbf{x}-\mathbf{b}, \varepsilon= \pm 1$.

Take the edge corresponding to $\lambda=\left(s, b^{(s)}, \varepsilon(l-1)\right)$ of level $l>0$ to be the maximum $s$-edge in $\beta(\lambda)$ if $\varepsilon=1$, or the minimum $s$-edge in $\beta(\lambda)$ if $\varepsilon=-1$. (If $l=1$ we can take either the maximum or the minimum $s$-edge; for definiteness, we choose the maximum $s$-edge.) Then the conditions of our general result above are satisfied, so $\mathscr{L}$ is equivalent to a 2-complex $\mathscr{L}_{0}$ with edge set

where

$$
\left\{b_{i}^{ \pm 1}: b \in \mathbf{b}, i \in \mathbb{Z}\right\} \cup \mathbf{s},
$$

$$
s=\left\{s_{0}^{ \pm 1}:\left\{s, b^{(s)}\right\} \text { a red edge }\right\} \cup\left\{s_{0}^{ \pm 1}, s_{1}^{ \pm 1}:\left\{s, b^{(s)}\right\} \text { a green edge }\right\},
$$

and defining paths $\gamma(\lambda)$ where $\lambda$ ranges over the elements (8), (9), (10). If $\lambda$ is an element in (10) then $\gamma(\lambda)=\beta(\lambda)$.

To obtain a further equivalence, choose an extremal vertex of the tree $\Gamma$. By relabelling vertices if necessary, we may assume that this extremal vertex is 1 . Let $\{1, p\}$ be the unique edge of $\bar{\Gamma}$ incident with 1 . Define an index $\lambda$ of $\mathscr{L}_{0}$ to have:

level 0 if $\lambda$ is an element (8), (9), or is an element (10) not involving $b(1)$;

level $l(l>0)$ if $\lambda=(b(1), b(p), \varepsilon(l-1)), \varepsilon= \pm 1$.

Take the edge corresponding to $\lambda=(b(1), b(p), \varepsilon(l-1))$ of level $l>0$ to be the maximum $b(1)$-edge in $\beta(\lambda)$ if $\varepsilon=1$ or if $l=1$, and the minimum $b(1)$-edge in $\beta(\lambda)$ if $\varepsilon=-1$ and $l>1$. Then by our general result above, $\mathscr{L}_{0}$ is equivalent to a 2 -complex $\mathscr{L}_{1}$ with edge set

$$
\left\{b(1)_{0}^{ \pm 1}, b(1)_{1}^{ \pm 1}\right\} \cup\left\{b_{i}^{ \pm 1} ; b \in \mathbf{b}-\{b(1)\}, i \in \mathbb{Z}\right\} \cup \mathbf{s},
$$

and defining paths $\delta(\lambda)$ where $\lambda$ ranges over the elements (8), (9), and the elements (10) not involving $b(1)$. If $\lambda$ is an element (10) not involving $b(1)$ then $\delta(\lambda)=\gamma(\lambda)=\beta(\lambda)$.

Now consider the tree $\bar{\Gamma}-\{1,\{1, \mathrm{p}\}\}$. We can assume (relabelling vertices if necessary) that 2 is an extremal vertex of this tree, and we let $\{2, q\}$ be the unique edge in this tree incident with 2 . Define an index $\lambda$ of $\mathscr{L}_{1}$ to have:

level 0 if $\lambda$ is an element (8), (9), or is an element (10) not involving $b(1)$ or $b(2)$; level $l(l>0)$ if $\lambda=(b(2), b(q), \varepsilon(l-1)), \varepsilon= \pm 1$. 
Then an argument like that in the previous paragraph shows that $\mathscr{L}_{1}$ is equivalent to a 2-complex with edge set

$$
\left\{b(1)_{0}^{ \pm 1}, b(1)_{1}^{ \pm 1}, b(2)_{0}^{ \pm 1}, b(2)_{1}^{ \pm 1}\right\} \cup\left\{b_{i}^{ \pm 1}: b \in \mathbf{b}-\{b(1), b(2)\}, i \in \mathbb{Z}\right\} \cup \mathbf{s},
$$

and defining paths indexed by the elements (8), (9), and the elements (10) not involving $b(1), b(2)$.

Continuing this procedure, we eventually arrive at a 2-complex with edge set

$$
\left\{b(m)_{0}^{ \pm 1}, b(m)_{1}^{ \pm 1}: 1 \leqslant m \leqslant r-1\right\} \cup\left\{b(r)_{i}^{ \pm 1}: i \in \mathbb{Z}\right\} \cup s,
$$

and defining paths indexed by the elements (8), (9). The edges $\left\{b(r)_{i}^{ \pm 1}: i \in \mathbb{Z}\right\}$ give rise to a maximal subtree of this complex. Collapsing this maximal subtree then gives a finite presentation.

\section{APPENDIX}

\section{Geometric aspects of combinatorial group theory}

A 1-complex $\mathscr{X}$ consists of two disjoint sets $V$ (vertices), $E$ (edges) together with three functions $\iota: E \rightarrow V, \tau: E \rightarrow V,,^{-1}: E \rightarrow E$ satisfying: $\iota\left(e^{-1}\right)=\tau(e),\left(e^{-1}\right)^{-1}=e, e^{-1} \neq e$ for all $e \in E$. A non-empty path $\alpha$ in $\mathscr{X}$ is a sequence $e_{1} e_{2} \ldots e_{n}(n \geqslant 1)$ of edges with $\tau\left(e_{i}\right)=\iota\left(e_{i+1}\right)(1 \leqslant i<n)$. We define $\iota(\alpha), \tau(\alpha)$ to be $\iota\left(e_{1}\right), \tau\left(e_{n}\right)$ respectively. The path is said to be closed if $\iota(\alpha)=\tau(\alpha)$. The path is said to be reduced if $e_{i+1} \neq e_{i}^{-1}(1 \leqslant i<n)$. The inverse $\alpha^{-1}$ of $\alpha$ is the path $e_{n}^{-1} \ldots e_{2}^{-1} e_{1}^{-1}$.

With each vertex $v$ of $\mathscr{X}$ we associate an empty path $1_{v}$ (or simply 1 ). This path has no edges. Moreover, $\iota\left(1_{v}\right)=\tau\left(1_{v}\right)=v$ and $1_{v}^{-1}=1_{v}$.

We say that the product $\beta \gamma$ of two paths $\beta, \gamma$ is defined if $\tau(\beta)=\iota(\gamma)$. Then $\beta \gamma$ is the path consisting of the edges of $\beta$ followed by the edges of $\gamma$. We have $\iota(\beta \gamma)=\iota(\beta)$, $\tau(\beta \gamma)=\tau(\gamma)$.

A 1-complex is said to be connected if, given any two vertices $u, v$, there is a path $\alpha$ with $\iota(\alpha)=u, \tau(\alpha)=v$. A 1-complex with a single vertex is called a bouquet. A connected 1-complex is said to be a tree if it has no non-empty reduced closed paths. For a 1-complex $\mathscr{L}$, a maximal element of $\{\mathscr{T}: \mathscr{T}$ is a tree contained in $\mathscr{X}\}$ (which exists by Zorn's lemma) is called a maximal subtree of $\mathscr{X}$.

Let $\mathscr{X}, \mathscr{Y}$ be 1-complexes. A mapping (of 1-complexes) $\phi: \mathscr{X} \rightarrow \mathscr{Y}$ is a function sending vertices of $\mathscr{X}$ to vertices of $\mathscr{Y}$, and paths in $\mathscr{X}$ to paths in $\mathscr{Y}$, and satisfying: $\phi\left(1_{v}\right)=1_{\phi(v)}$ for all vertices $v$ of $\mathscr{X} ; \phi\left(\alpha^{-1}\right)=\phi(\alpha)^{-1}$ for all paths $\alpha$ in $\mathscr{X}$; whenever $\alpha_{1} \alpha_{2}$ is defined $\left(\alpha_{1}, \alpha_{2}\right.$ paths in $\left.\mathscr{X}\right) \phi\left(\alpha_{1}\right) \phi\left(\alpha_{2}\right)$ is also defined, and $\phi\left(\alpha_{1} \alpha_{2}\right)=\phi\left(\alpha_{1}\right) \phi\left(\alpha_{2}\right)$.

A 2-complex $\mathscr{K}$ is an object $\langle\mathscr{X} ; \beta(\lambda)(\lambda \in \Lambda)\rangle$ where $\mathscr{X}$ is a 1 -complex (called the 1 -skeleton of $\mathscr{K})$ and each $\beta(\lambda)$ is a closed path in $\mathscr{X}$. The $\beta(\lambda)$ are called defining paths. The elements of $\Lambda$ are called indices. The 2-complex $\mathscr{K}$ is said to be connected if $\mathscr{X}$ is connected. If $\mathscr{X}$ is a bouquet then $\mathscr{K}$ is called a presentation.

We define an equivalence relation $\sim: \mathcal{K}$ (or simply $\sim$ ) on paths in $\mathscr{K}$ as follows. An 
elementary reduction of a path $\alpha$ is a transformation of $\alpha$ to $\alpha_{1} \alpha_{2}$ if $\alpha$ has one of the forms $\alpha_{1} \gamma \gamma^{-1} \alpha_{2}, \alpha_{1} \beta(\lambda)^{\varepsilon} \alpha_{2}(\lambda \in \Lambda, \varepsilon= \pm 1)$. A pair of paths is called a reduction pair if one of the paths is obtained from the other by an elementary reduction. Then for two paths $\alpha, \alpha^{\prime}$, we define $\alpha \sim_{\mathscr{K}} \alpha^{\prime}$ if and only if there is a sequence of paths $\alpha=$ $\alpha_{0}, \alpha_{1}, \alpha_{2}, \ldots, \alpha_{m}=\alpha^{\prime}$ with $\left\{\alpha_{i}, \alpha_{i+1}\right\}$ a reduction pair for $i=0, \ldots, m-1$. The $\sim$-equivalence class containing $\alpha$ is denoted by $[\alpha]_{\mathscr{H}}$ (or simply by $[\alpha]$ ). A path which is $\sim$-equivalent to an empty path is said to be contractible. (We also say that such a path is a consequence of the paths $\beta(\lambda)(\lambda \in \Lambda)$.)

Contractible paths can be looked at pictorially using van Kampen diagrams. These are discussed in [8, Chapter V] for presentations, but the theory for 2-complexes (or even generalized 2-complexes) is entirely similar [6]. Put briefly and roughly, if one has a tessellation of the surface of a sphere with each region except one having its boundary labelled by an element of $\{\beta(\lambda): \lambda \in \Lambda\}$, then the boundary label of the exceptional region is contractible.

For a fixed vertex $v$ of $\mathscr{K}$, the fundamental group $\pi_{1}(\mathscr{K}, v)$ has underlying set

$$
\{[\gamma]: \iota(\gamma)=\tau(\gamma)=v\}
$$

and binary operation $\left[\gamma_{1}\right]\left[\gamma_{2}\right]=\left[\gamma_{1} \gamma_{2}\right]$. If $\mathscr{K}$ is connected then the isomorphism type of this group is independent of the choice of $v$. We can then refer to the fundamental group of $\mathscr{K}$, which we denote by $\pi_{1}(\mathscr{K})$.

Suppose that $\mathscr{H}$ is connected and that $H$ is a subgroup of $\pi_{1}(\mathscr{K})$. Then we can construct from $\mathscr{K}$ another connected 2-complex $\mathscr{\mathscr { H }}$ (called the covering of $\mathscr{K}$ corresponding to $H$ ) with $\pi_{1}(\tilde{\mathscr{K}}) \cong H$. (We have no need to describe the general construction here.)

Let $\mathscr{K}, \mathscr{L}$ be 2 -complexes. A mapping (of 2-complexes) $\phi: \mathscr{K} \rightarrow \mathscr{L}$ is a mapping of 1 -complexes from the 1-skeleton of $\mathscr{K}$ to the 1-skeleton of $\mathscr{L}$ such that the image of each contractible path in $\mathscr{K}$ is a contractible path in $\mathscr{L}$. We say that $\phi$ is an equivalence if there is another mapping $\psi: \mathscr{L} \rightarrow \mathscr{K}$ such that

$$
\psi \phi(\alpha) \sim_{\mathscr{K}} \alpha
$$

for each path $\alpha$ in $\mathscr{K}$,

$$
\phi \psi(\beta) \sim_{\mathscr{L}} \beta
$$

for each path $\beta$ in $\mathscr{L}$. Two 2-complexes are said to be equivalent if there is an equivalence between them.

We also have the idea of a based mapping $\phi:(\mathscr{K}, u) \rightarrow(\mathscr{L}, \phi(u))$. Here $u$ is a vertex of $\mathscr{K}$ and $\phi$ is a mapping from $\mathscr{K}$ to $\mathscr{L}$. We say that $\phi$ is a based equivalence if there is a based mapping $\psi:(\mathscr{L}, \phi(u)) \rightarrow(\mathscr{K}, u)$ such that (11) and (12) hold for all paths $\alpha, \beta$ with $\iota(\alpha)=\tau(\alpha)=u, \iota(\beta)=\tau(\beta)=\phi(u)$. Obviously an equivalence gives rise to a based equivalence for any choice of $u$. If $\phi:(\mathscr{K}, u) \rightarrow(\mathscr{L}, \phi(u))$ is a based equivalence then $\pi_{1}(\mathscr{K}, u) \cong \pi_{1}(\mathscr{L}, \phi(u))$ (an isomorphism being given by $[\gamma]_{\mathscr{K}} \rightarrow[\phi(\gamma)]_{\mathscr{L}},[\gamma]_{\mathscr{K}} \in \pi_{1}(\mathscr{K}, u)$ ).

Given a connected 2-complex $\mathscr{K}=\langle\mathscr{X} ; \beta(\lambda)(\lambda \in \Lambda)\rangle$ and a vertex $u$, there is a based equivalence from $(\mathscr{K}, u)$ to a presentation, as follows. Let $\mathscr{T}$ be a maximal subtree of $\mathscr{X}$, and let $f^{ \pm 1}(f \in \mathbf{f})$ be the edges of $\mathscr{X}$ lying outside $\mathscr{T}$. Let $\mathscr{B}$ be the bouquet with vertex $v$ 
and edges $\bar{f}^{ \pm 1}(f \in \mathbf{f})$. Define $\phi: \mathscr{X} \rightarrow \mathscr{B}$ by

$$
\phi(e)= \begin{cases}1 & e \in \mathscr{T} \\ \bar{f}^{\varepsilon} & e=f^{\varepsilon}(f \in \mathbf{f}, \varepsilon= \pm 1) .\end{cases}
$$

Let $\mathscr{P}=\langle\mathscr{B} ; \phi(\beta(\lambda))(\lambda \in \Lambda)\rangle$. Then $\phi:(\mathscr{K}, u) \rightarrow(\mathscr{P}, v)$ is a based equivalence. We say that $\mathscr{P}$ is obtained from $\mathscr{K}$ by collapsing the maximal subtree $\mathscr{T}$.

Finally we discuss homology. Consider a connected 2-complex

$$
\mathscr{K}=\langle\mathscr{X} ; \beta(\lambda)(\lambda \in \Lambda)\rangle \text {. }
$$

Let $E^{+}$consist of exactly one of $e, e^{-1}$ for each pair of edges $\left\{e, e^{-1}\right\}$ of $\mathscr{X}$. Let $C_{1}$ be the free abelian group on $E^{+}$(the group of 1-chains) and let $C_{0}$ be the free abelian group on the vertex set of $\mathscr{X}$. There is a homomorphism $\partial: C_{1} \rightarrow C_{0}$ defined by $\partial(e)=\tau(e)-\iota(e)\left(e \in E^{+}\right)$. The kernel of this homomorphism is the group $Z_{1}$ of 1-cycles. Now let $C_{2}$ be the free abelian group on $\Lambda$. There is a homomorphism $\partial: C_{2} \rightarrow C_{1}$ defined by $\partial(\lambda)=\widehat{\beta(\lambda)}$, where, for a path $\alpha=e_{1}^{\varepsilon_{1}} e_{2}^{\varepsilon_{2}} \ldots e_{m}^{\varepsilon_{m}}\left(e_{i} \in E^{+}, \varepsilon_{i}= \pm 1 \quad(1 \leqslant i \leqslant m)\right)$, $\hat{\alpha}=\varepsilon_{1} e_{1}+\varepsilon_{2} e_{2}+\ldots+\varepsilon_{m} e_{m}$. The group $\operatorname{Im} \partial=B_{1}$ is called the group of 1-boundaries. We have $B_{1} \leqslant Z_{1}$. The quotient $Z_{1} / B_{1}$ is the first integral homology group $H_{1}=H_{1}(\mathscr{K} ; \mathbb{Z})$ of $\mathscr{K}$. It can be shown [14, p. 46] that $H_{1}$ is isomorphic to the abelianization $\pi_{1}(\mathscr{K})^{\mathrm{ab}}$ of $\pi_{1}(\mathscr{K})$.

\section{REFERENCES}

1. E. Artin, Theorie der Zöpfe, Abh. Math. Sem. Univ. Hamburg 4 (1925), 47-72.

2. J. S. Birman, Braids, Links and Mapping Class Groups (Annals of Mathematics Studies 82, Princeton University Press, 1974).

3. B. Bollobás, Graph Theory (Graduate Texts in Mathematics 63, Springer-Verlag, 1979).

4. D. Cohen, Combinatorial Group Theory: A Topological Approach (Queen Mary College Mathematics Notes).

5. E. A. Gorin and V. Ja. Lin, Algebraic equations with continuous coefficients and some problems of the algebraic theory of braids, Math. USSR-Sb. 7 (1969), 569-596.

6. J. Howie and S. J. Pride, A spelling theorem for staggered generalized 2-complexes, with applications, Invent. Math. 76 (1984), 55-74.

7. D. L. Johnson, Analogues of the braid group, Proceedings of the Conference GroupsKorea 1983 (Lecture Notes in Mathematics 1098, Springer-Verlag, 1984).

8. R. C. Lyndon and P. E. Schupp, Combinatorial Group Theory (Springer-Verlag, 1977).

9. W. Magnus, Braid groups: a survey, Proceedings of the Second International Conference on the Theory of Groups (Lecture Notes in Mathematics 372, Springer-Verlag, 1974).

10. A. K. Napthine, Analogues of the braid group whose graphs are stars, Proc. Edinburgh Math. Soc. (2) 28 (1985), 35-39.

11. S. J. Pride, Equivalences of combinatorial 2-complexes, in preparation.

12. J-P. Serre, Trees (Springer-Verlag, 1980).

13. J. Stillwell, Classical Topology and Combinatorial Group Theory (Graduate Texts in Mathematics 72, Springer-Verlag, 1980).

14. H. Zieschang, E. Vogt and H.-D. Coldewey, Surfaces and Planar Discontinuous Groups (Lecture Notes in Mathematics 835, Springer-Verlag, 1980).

Minster SCHOOL

NOTTINGHAM ROAD

SOUTHWELL NG25 OMG
UNIVERSITY OF GLASGOW DepartMENT OF MATHEMATiCS UNIVERSITY GARDENS GLASGOW G12 8QW 